\title{
Artificial Bee Colony and Its Application for Image Fusion
}

\author{
Prabhat Kumar Sharma, Bhavya V S,Nayyashree K M, Sunil K S, Pavithra P \\ Dept. of ISE, Reva Institute of Technology and Management, Yelahanka, Bangalore- 560 064, India \\ (prabhat19sharma, bhavyav.sridhar, navyashreekm26, sunhill.ise, pavithraperumal)@gmail.com
}

\begin{abstract}
Artificial Bee Colony (ABC) is one of the latest $\mathrm{s}$ warm algorith $\mathrm{m}$ based on the intelligent foraging behavior of honey bees introduced in the year 2005 by Karaboga since then it has been used for optimization of various solutions. And it is recently introduced for processing and analysis of images such as segmentation, object recognition and image retrieval. Fusing images from a vast collection of different images has become one of the interesting challenges and has drawn the attention of researchers towards the development of fusion techniques. In this paper, we have proposed the usage of $\mathrm{ABC}$ for optimal fusion of multi-temporal images and studied the effect of variation in the source area.
\end{abstract}

Index Terms - ABC, Image Fusion, Quality measure

\section{Introduction}

Image fusion is the process of combining information from two or more images of a scene into a single composite image that is more informative and is more suitable for visual perception or computer processing. The goal of image fusion is to integrate complementary multi-sensor, multi-temporal and/or multi-view information into one new image[1].Image fusion is used in the field of image classification, aerial and satellite imaging[2], medical imaging[3], robot vision, concealed weapon detection[4], multi-focus image fusion [5], face recognition.

Fusing images from a vast collection of different images has become one of the interesting challenges and has drawn the attention of researchers towards the development of fusion techniques. A large number of image fusion techniques proposed were based on wavelet transformation. Image fusion using the DTCWT was proposed in[6]. Image fusion based on a type of shift-invariant DWT was suggested in [7]. Information at higher levels of abstraction such as image edges and image segment boundaries are used to guide image fusion by pyramids [8].A new image fusion method that combines HIS transform and curvlet transform was proposed in [9].

In recent time many work has been done to introduce this $\mathrm{ABC}$ algorithm is field of image processing. In image retrieval system $\mathrm{ABC}$ algorithm is used to fuse similarity score based on color and texture features of an image thereby achieving very high classification accuracy and minimum retrieval time [10]. Image registration is a hot topic in the field of image processing, and it is widely used in various applications. A novel image reg is tration technique was proposed. For the model the ABC algorithm was used [11].Image segmentation is still a crucial problem in image processing. It hasn't yet been solved very well. ABC algorithm is used to achieve multi-level thresholding image segmentation based on PSNR[12].An improved $\mathrm{ABC}$ algorithm was introduced for the object recognition problem in complex digital images which resulted in very improved and efficient object recognition[13].Likewise many research work is going on to introduce this algorithm in the field of image processing. In the literature survey conducted by us we found that no work has been done in image fusion using $\mathrm{ABC}$ algorithm.

Many existing image fusion methods are based on wavelets and pyramid, which are very complex to implement. Some of the primitive image fusion techniques based on arithmetic operations on the pixels are simple. But these simple approaches often have serious side effects such as reducing or increasing the contrast[4].

Our proposed image fusion technique based on $\mathrm{ABC}$ is simple to implement and the quality of image obtained is better than that of the pixel-pixel arith metic operation.

The remainder of this paper is organized as follows: Section 2 describes $\mathrm{ABC}$ concept and our proposed method followed by the merits and demerits of our method. Section 3 describes implementation and performance parameter of our experiment. Section 4 presents the result and evaluation. Conclusion and future work are given in the final section.

\section{Proposed Work}

\subsection{ABC Concept}

$\mathrm{ABC}$ algorithm is based on the intelligent way of the bees interacting with each other. Honey bees being social insects divide their work among themselves: 
Employed bees, Onlooker bees and Scout Bees [14]. Their activities are categorized into four main phases: Initialization phase, Employed bee phase, Onlooker bee phase and Scout bee phase. In initialization phase, each employed bee is assigned with different food resources. In employed bee phase, each employed bee calculates the nectar amount of the food resource associated with it and the distance of it from the hive. After collecting the important information of the source the employed bee share the gathered information with the bees waiting in the hive. In onlooker bee phase, onlooker bees (the bees waiting in the hive) read information regarding different food resources and choose the best food resource. In scout bees phase, the employed bees whose food resource becomes abandoned turns into scout bee. The main job of scout bees is to search for new food resources.

In field of computer science and operation research, $\mathrm{ABC}$ is mainly used for solution of optimization problem. When related to optimization problem, the food resources are the set of different feasible solutions available. The nectar value of each food resource calculated by the employed bees is the fitness value of the a particular feasible solution[15]. The food resource chosen by the onlooker bees are the best optimal solution among the available set of feasible solution.

The main steps of the algorithm are given below[16]:

- Initialization

- Repeat

(a). Assign the employed bees onto the food sources in the memory;

(b). Place the onlooker bees on the food sources in the memory;

(c). Send the scouts to the search area for discovering new food sources.

- Until (requirements are met)

\subsection{Proposed ABC in image fusion}

The objective of the study is to apply ABC algorithm for fusion of multitemporal images.Mutitemporal images are the images taken at different time interval at same scene.

In our experiment, the initialization phase consists of assigning a source to the employed bees i.e we are reading two images. Since the work is on fusion of two images, each of these images is divided into small areas which become the source for employed bees i.e we are dividing images into small window. As we are interested only in the information contained in the individual images taken at different time intervals, we have chosen entropy as the nectar and the entropy value is the measure of the nectar amount i.e entropy of each corresponding window of two images is calculated. These values are compared by the onlooker bees and they choose the pixel of the image from the source which has the highest nectar amount and put them into the hive (fused image) i.e. entropy value of the corresponding windows of two images are compared with each other, the window having highest entropy value is chosen for output images. Further the centre pixel of the window is chosen for output image

\section{1) Methodology}

The methodology followed for conduction of the experiment is described in figure 1.

The arithmetic method of image fusion is performed by taking the average of corresponding pixels of two images and placing it in the corresponding position in the output image.

$$
\mathrm{C}[\mathrm{i}, \mathrm{j}]=(\mathrm{a}[\mathrm{i}, \mathrm{j}]+\mathrm{b}[\mathrm{i}, \mathrm{j}]) / 2
$$

Where $0<\mathrm{i}<\mathrm{m}$ is height and $0<\mathrm{j}<\mathrm{m}$ is weight of the window.

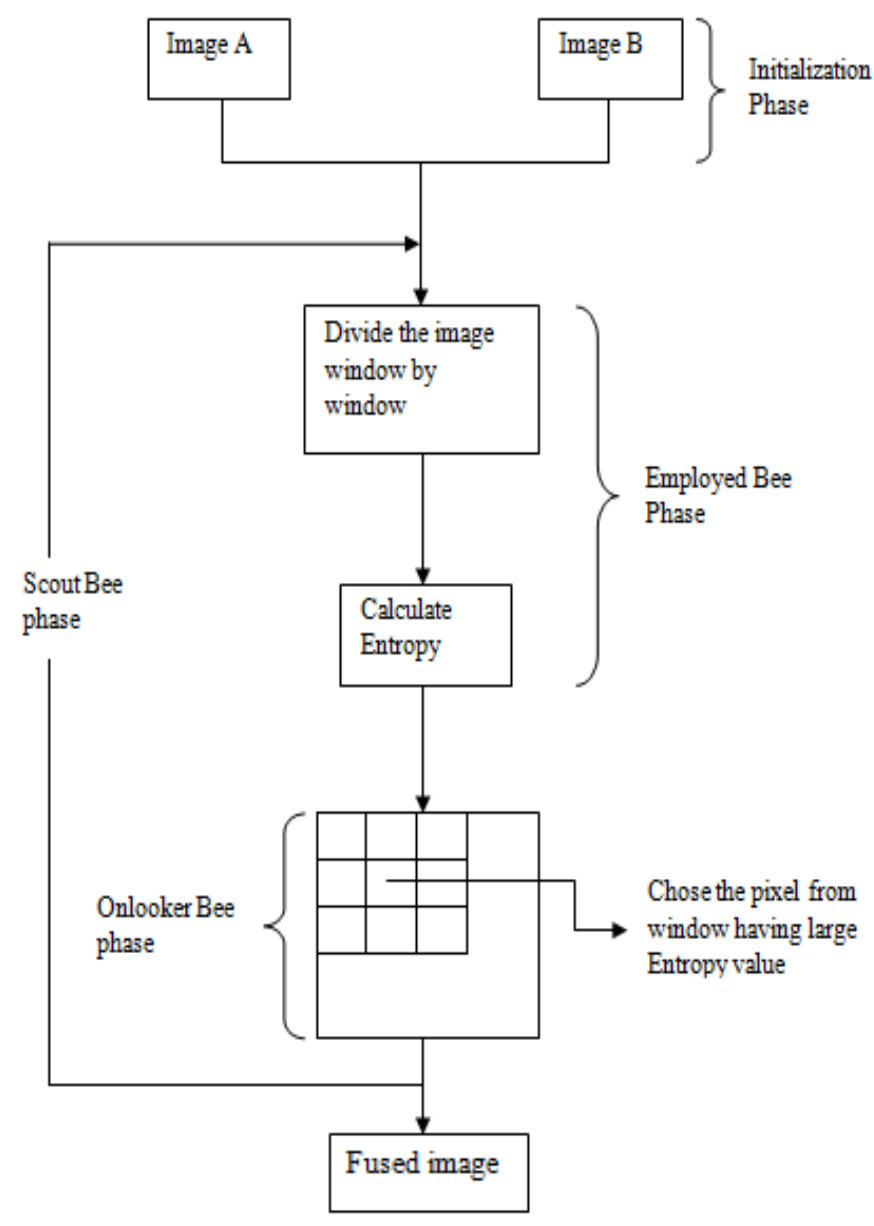

Fig. 1: Methodology

\section{2) Algorithm}

Step 1: Start.

Step 2: Initialization phase:

Read two images(Source). 
Step 3: Employed Bee phase:

Select a source area of size (m x m) in both the images.

Calculate the properties ( nectar amount) for both the source area.

Step 4: Onlooker Bee phase:

Select the centre pixel(nectar) of the source area(source) having highest property value (nectar value).

Store the selected pixel in 2D buffer(hive).

Step 5: Scout Bee phase:

Select next source area (new source) and repeat the steps 3 to $5,(\mathrm{p}-\mathrm{m} / 2) *(\mathrm{q}-\mathrm{m} / 2)$ times.

Here, $p=$ Height and $q=$ width of selected image and $\mathrm{w}=$ window size.

Step 6: Stop

\subsection{Advantages and Limitations}

The merit of the proposed algorithm is that it's easy to implement and the quality of image is better. The demerit of our work is the determination of window size. For the images chosen for the experiment larger window size gave better result but the same may not hold good for all the images.

\section{Implementation}

\subsection{Experimental setup}

The experiment was conducted using $\mathrm{VC}++6.0$ and the images were gray scale of the type RAW. The different types of images available for fusion were:

- Multi-view fusion of images from the same modality and taken at the same time but from different viewpoints.

- Multi-modal fusion of images coming from different sensors (visible and infrared, CT and MR, or panchromatic and mult ispectral satellite images).

- Multi-temporal fusion of images taken at different times in order to detect changes between them or to synthesize realistic images of objects which were not photographed in a desired time.

- Multi-focus fusion of images of a 3D scene taken repeatedly with various focal lengths.[17]

\subsection{Performance parameter}

The quality matrices for object analysis used for output image used in the experiment are[1]:

1. Entropy

2. Spatial frequency

\section{1) Entropy}

Entropy is a measure of the amount of information that can be derived from the image[6]. Entropy is calculated by the following equation:

$$
E=-\sum_{i=0}^{n-1} P(i) \log _{2} P(i)
$$

Where,

$\mathrm{n}=$ maximu $\mathrm{m}$ gray level considered

$\mathrm{P}(\mathrm{i})=$ normalized histogram of the graylevel $\mathrm{i}$.

By taking entropy into consideration we can merge multi-temporal images by extracting the best content available in different images.

\section{2) Spatial frequency}

Spatial frequency is the measure of the overall activity level in an image[6]. For an $\mathrm{MxN}$ image $\mathrm{F}$, with the gray value of pixel position $(m, n)$ denoted by $F(m$, $\mathrm{n})$, spatial frequency is computed as:

$$
S F^{2}=R F^{2}+C F^{2}
$$

Row Frequency $(\mathrm{RF})$ and Column Frequency $(\mathrm{CF})$ are given by the equations:

$$
\begin{aligned}
& R F=\sqrt{\frac{1}{M N} \sum_{m=1}^{M} \sum_{n=2}^{N}(F(m, n)-F(m, n-1))^{2}} \\
& C F=\sqrt{\frac{1}{M N} \sum_{n=1}^{N} \sum_{m=2}^{M}(F(m, n)-F(m-1, n))^{2}}
\end{aligned}
$$

\section{IV. results and discussion}

\subsection{Fused Images}

The results of images fused by using $\mathrm{ABC}$ and the arithmetic based fusion are given below in figure 2,3 and 4.

We have chosen three different sets of multi te mporal images for our experimental study. 


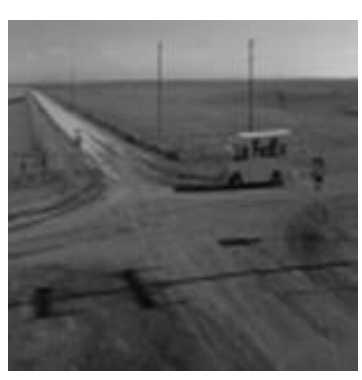

(a)

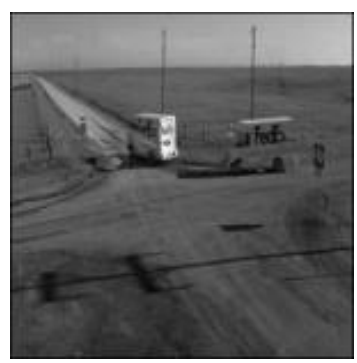

(c)

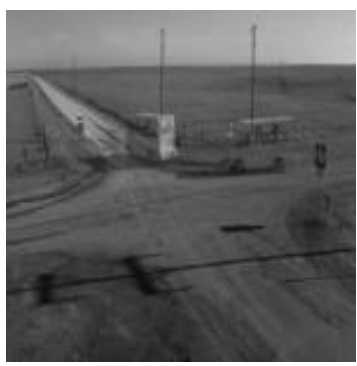

(e)

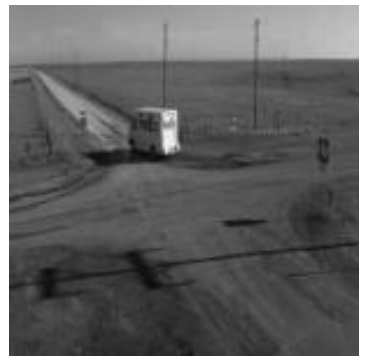

(b)

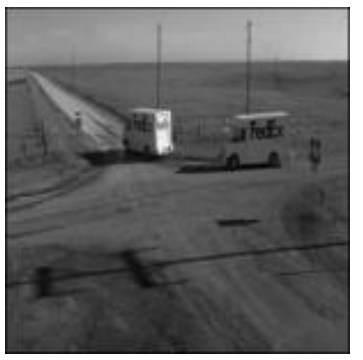

(d)

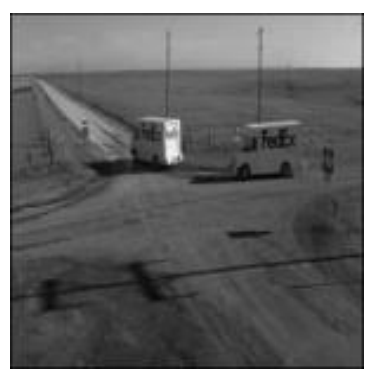

(f)

Fig. 2: Cab, Input images used for fusion-(a),(b); Images fused using ABC with varying source areas-(c),(d),(e);

(c)-Source area $=11 \times 11$,(d)-Source area $=37 \times 37$, (e)- Source area $=47 \times 47$,(f)-Image fused using average method.

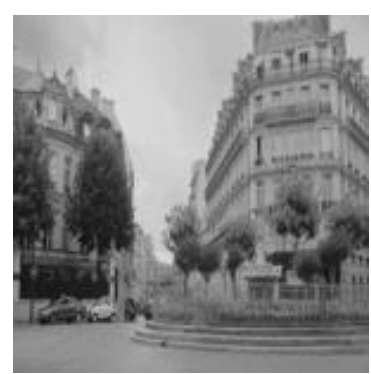

(a)

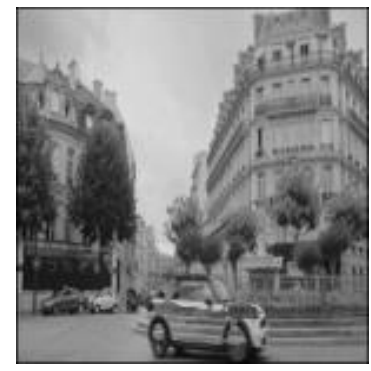

(c)

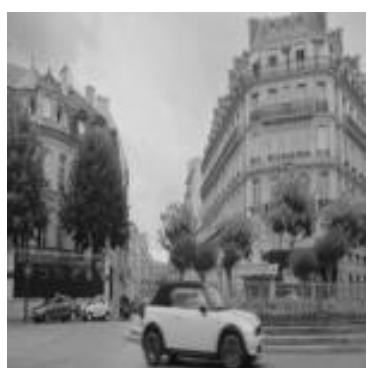

(b)

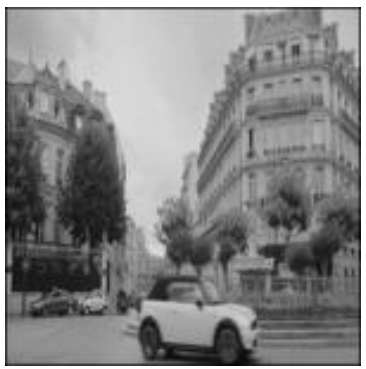

(d)

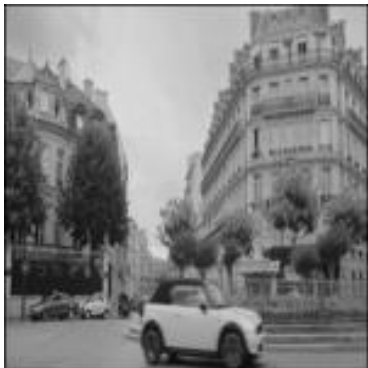

(e)

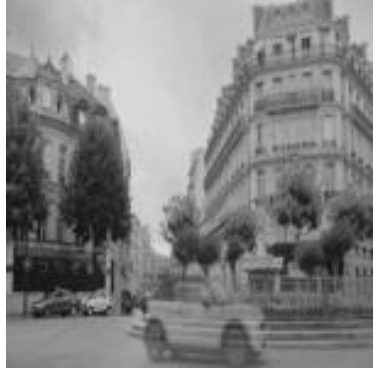

(f)

Fig. 3:Car input images used for fusion-(a),(b); Images fused using ABC with varying source area-(c),(d),(e); (c)-Source area $=11 \times 11,(d)$-Source arear $=37 \times 37$,

(e)-Source area $=47 \times 47$,(f)-Image fused using average method.

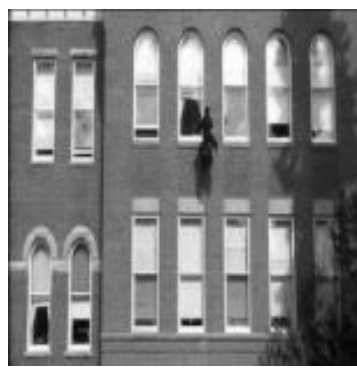

(a)

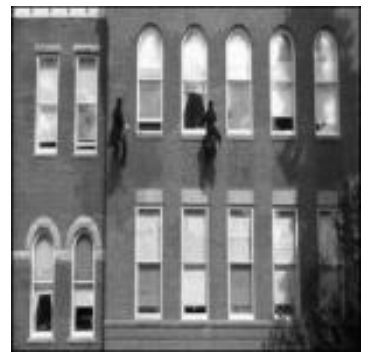

(c)

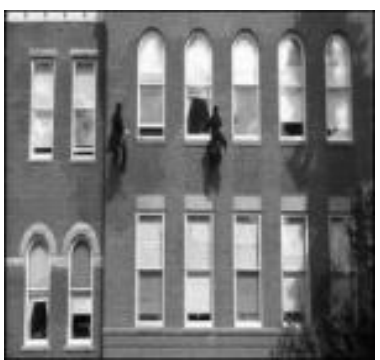

(e)

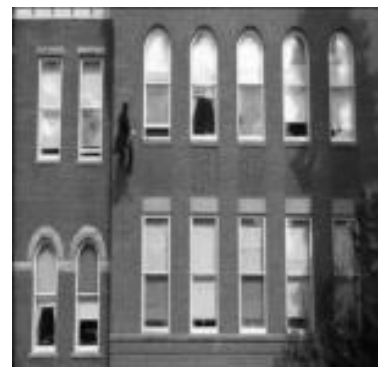

(b)

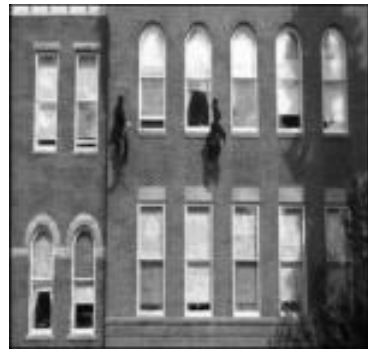

(d)

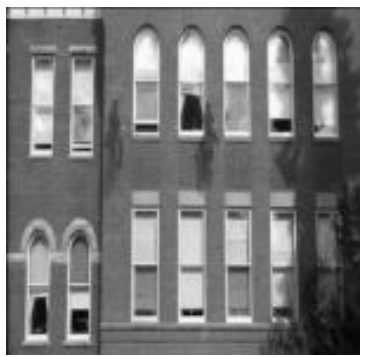

(f)

Fig. 4: Man input images used for fusion-(a),(b); Images fused using ABC with varying source area -(c),(d),(e); (c)-Source area $=11 \times 11,(d)-$ Source area $=37 \times 37$,

(e)- Source area $=47 \times 47$,(f)-Image fused using average method

\subsection{Analysis of entropy}

All the output images obtained were subjected to objective and subjective quality analysis described in [1]. 
The following graph shows the analysis of the entropy of the different output images obtained Entropy(Cab)

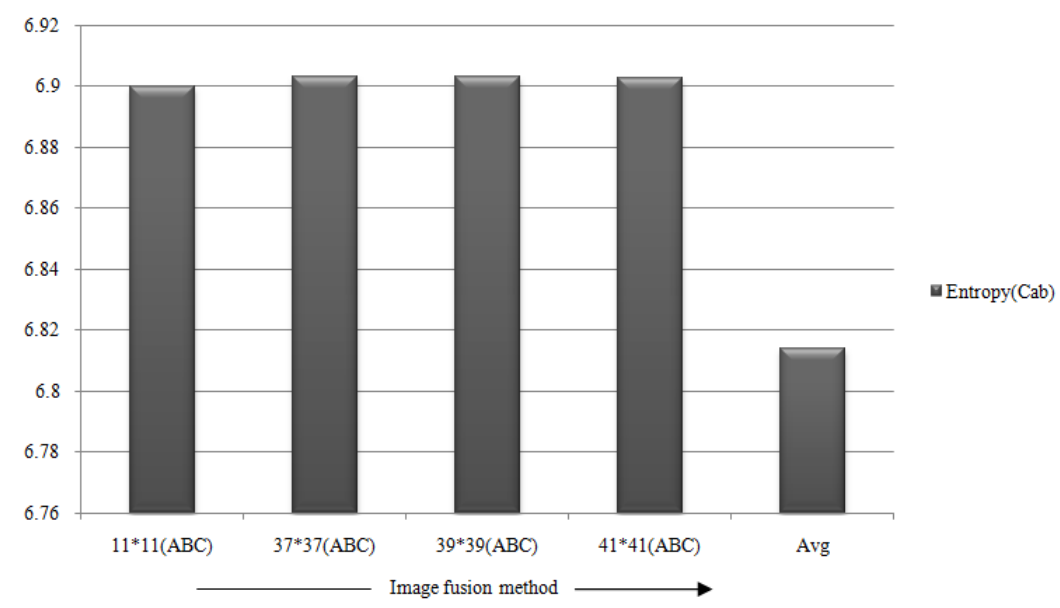

Graph I: Cab; Entropy values of the output image using $\mathrm{ABC}$ and average method of different source area

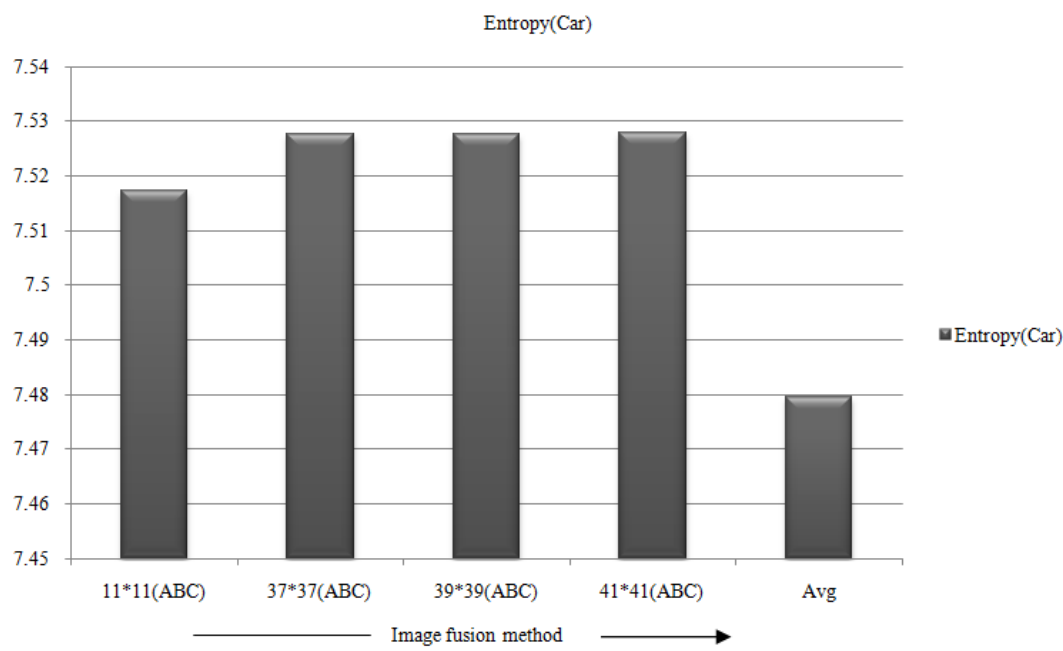

Graph II: Car;Entropy values of the output image using $\mathrm{ABC}$ and average method of different source area.

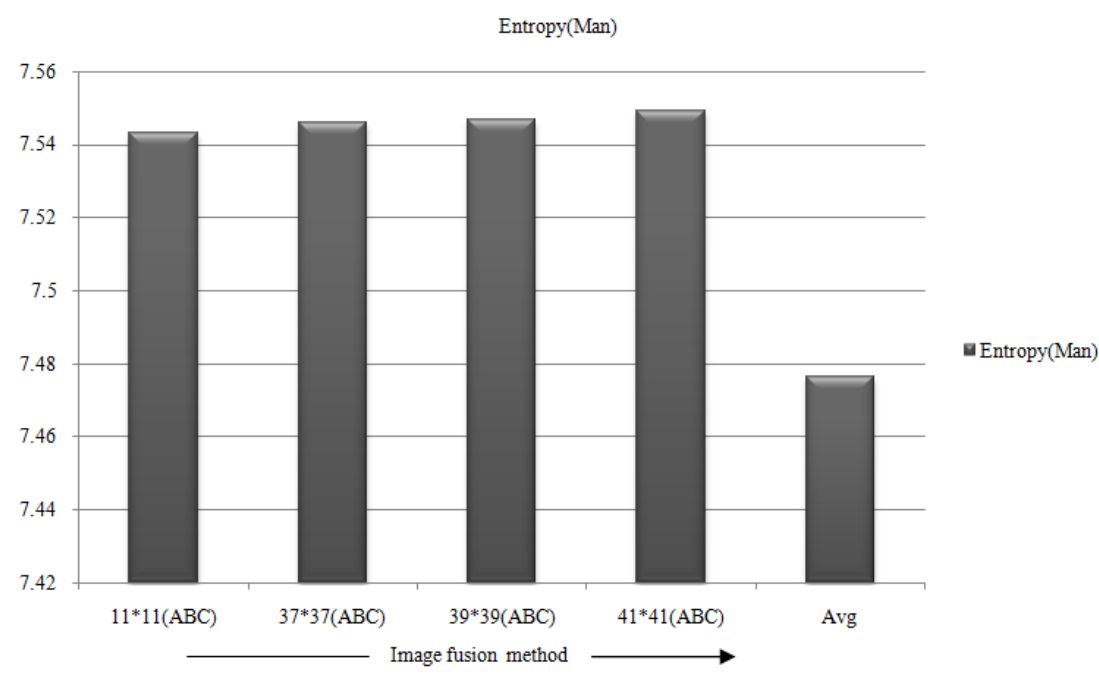

Graph III: Man; Entropy values of the output image using ABC and average method of different source area.

The maximu $m$ value of entropy of an image is 8 and the image with this value is said to have the maximum amount of information. Hence higher the value of entropy, better is the quality of the image [1]. The 
graphs I, II and III indicate that the images fused by $\mathrm{ABC}$ has higher entropy value hence is better than that of average method.

\subsection{Analysis of spatial frequency}

The following graph shows the analysis of the entropy of the different output images obtained.

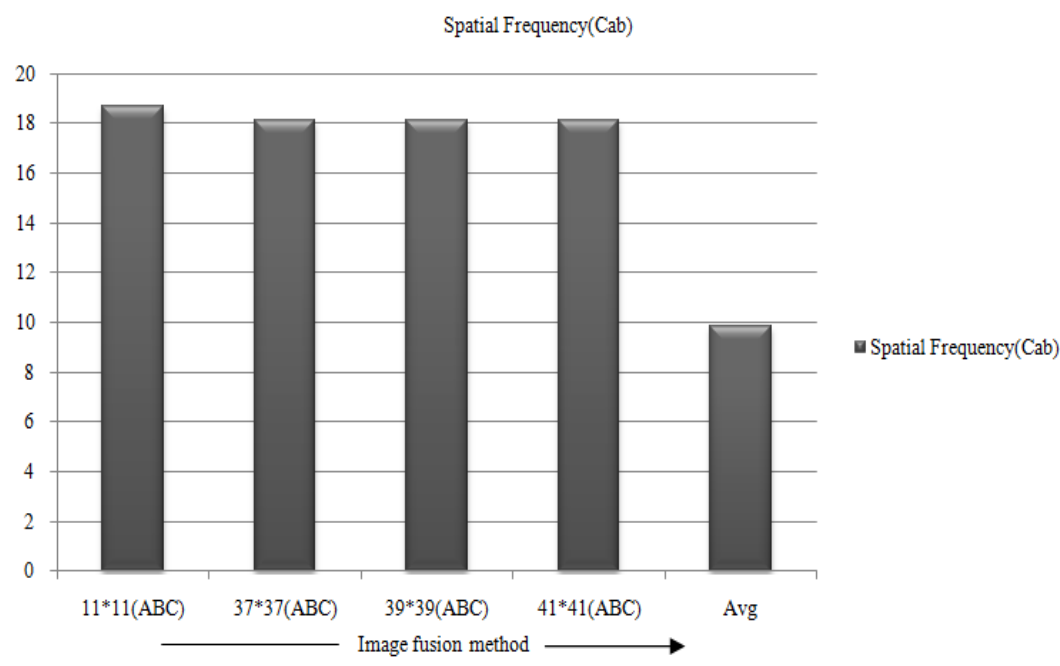

Graph IV: Cab; Saptial frequency values of the output image using ABC and average method of different source area

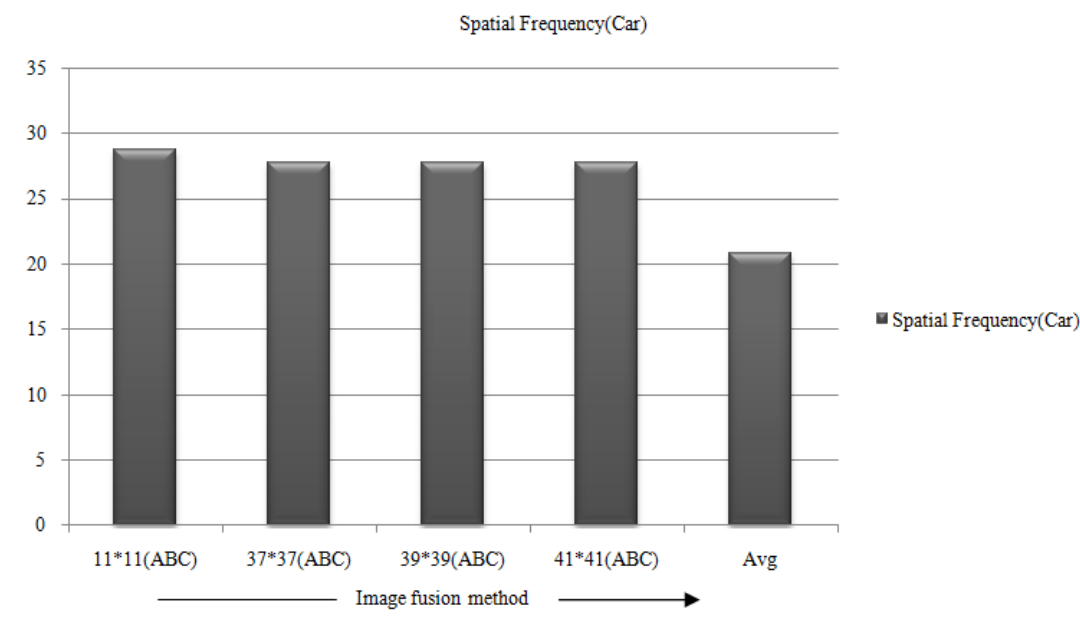

Graph V: Car; Spatial frequecny values of the output image using ABC aand average method of different source area

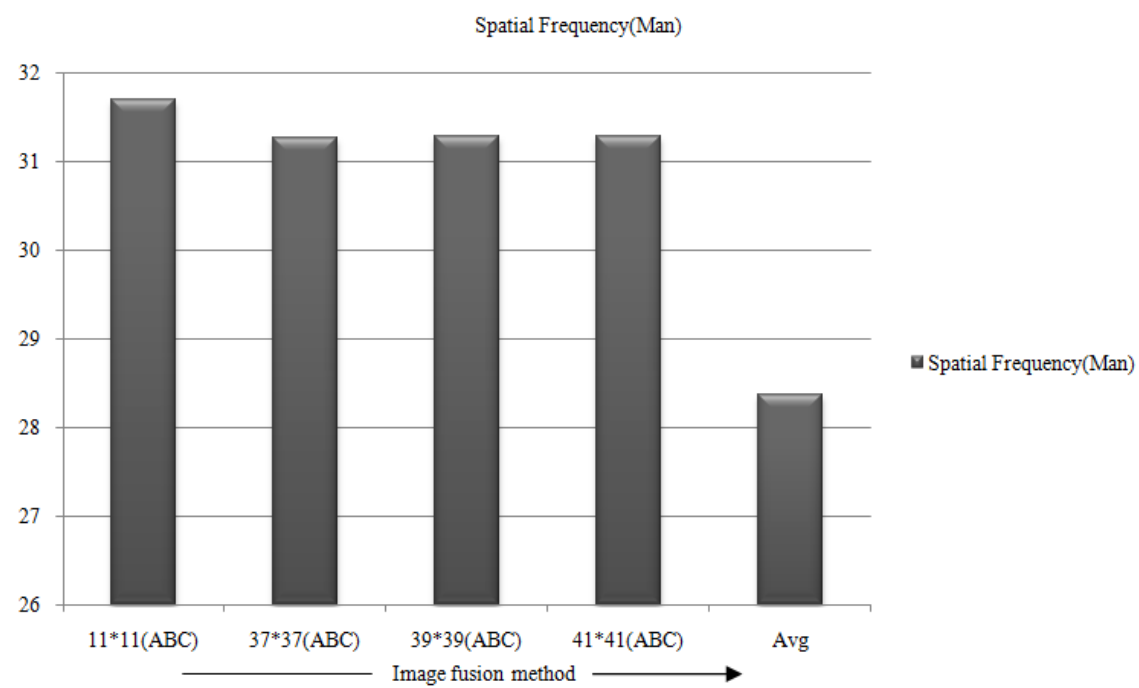

Graph VI: Man; Spatial frequecny values of the output image using ABC and average method of different source area 
Image with higher SF value indicates that the image have greater activity level, hence has better quality [1]. The graphs I, II and III indicate that the images fused by $\mathrm{ABC}$ has higher SF value hence is better than that of average method.

\section{Conclusion}

The application of $\mathrm{ABC}$ for image fusion was successful and the output images fused based on ABC has more information content (higher Entropy value) and also looks better (higher Spatial Frequency values) than the images fused by arithmetic operation, figure 2,3 and 4 . Although not much of variation was observed in the objective image quality metric values, a large amount of artifacts were observed by subjective analysis in the images which were fused using smaller source area than those of larger source areas.

Future work would include finding an optimized source area (window size) for fusion of images using $\mathrm{ABC}$.

\section{References}

[1] Ms. Shruthi T V, Ms. Ramyashree N, Ms. Pavithra P, Ms. Kamalam Balasubramani; "Analysis of Image Quality Using Quantitative Methods"; ICICES, $23^{\text {rd }}-24^{\text {th }}$ Feb 2011.

[2] Uttam Kumar, Chiranjit Mukhopadhyay and T. V. Ramachandra; "Pixel based fusion using IKONOS imagery", International Journal of Recent Trends in Engineering Vol 1, No. 1,2009.

[3] Wang Anna, Wu Jie, Li Dan, Chen Yu; " Research on Medical Image Fusion Based on Orthogonal Wavelet Packets Transformation Combined with 2v-SVM ", Complex Medical Engineering, 2007. CME 2007, ISBN: 978-1-4244-1078-1 Page no. 670-675. IEEE/ICME International Conference on 23-27 May 2007.

[4] Z. Xue, R S. Blum, and Y. Li; " Fusion of Visual and IR Images for Concealed Weapon Detection", Information Fusion, 2002. Proceedings of the Fifth IEEE International Conference on 2002, Vol 2, Page no: 1198 - 1205 .

[5] Muhammad Hassan Arif and Syed Sqlain Shah;" Block Level Multi-Focus Image Fusion using Wavelet Transform", Signal Acquisition and Processing, 2009. ICSAP 2009. International Conference on 3-5 April 2009, Page no: 213 - 216

[6] Paul Hill, Nishan Canagarajah and Dave Bull;" Image Fusion using ComplexWavelets ", BMVC Sept-2002, Page no: 487-496.

[7] Zhong Zhang."A Categorization of MultiscaleDecomposition-Based Image Fusion Schemes with a Performance Study for a Digital Camera
Application", Proceedings of the IEEE Aug 1999, Volume: 87, Is sue no: 8 Page no: 1315 - 1326.

[8] Vladimir Petrović;" Multi-level Image Fusion", www.imagefusion.org.

[9] Man wang, Dai-yang cao, Jie-lin zang,"Fusion of multispectral and panchromatic satellite images based on HIS and curvlet transformation", ICWAPR-2007,Vol:1 Page no: 321-325.

[10] D.Chandrakala and S.Sumathi;" Application of Artificial Bee Colony Optimization Algorithm for Image Classification Using Color and Texture Feature Similarity Fusion"; SRN Artificial Intelligence; Volume 2012 (2012), Article ID 426957.

[11] Shuihua Wang," Artificial Bee Colony used for Rigid Image Registration", International Journal of Research and Reviews in Soft and Intelligent Computing (IJRRSIC) Vol. 1, No. 2, June 2011.

[12] Cao Yun-Fei, Xiao Yong-Hao, Yu Wei-Yu and Chen Yong-Chang," Multi-level Threshold Image Segmentation Based on PSNR using Artificial Bee Colony Algorithm", China Research Journal of Applied Sciences, Engineering and Technology Published: January 15, 2011.

[13] Chidambaram Chidambaram, "An Improved Artificial Bee Colony Algorithm for the Object Recognition Problem in Complex Digital Images using Template Matching”, IJNCR, Vol 1,Issue 2,2010 .

[14] Adil Baykaso lu, Lale Özbakır and Pınar Tapkan, "Artificial Bee Colony Algorithm and Its Application to Generalized Assignment Problem", ISBN 978-3-902613-09-7, pp. 532, December 2007.

[15] A Survey on Artificial Bee Colony Algorithm, Karnan Marcus , KamalamBalasubramani, Department of Computer Science and Engineering, Tamil Nadu College of Engineering, Coimbatore, India Department of Information Science and Engineering, Atria Institute of Technology, Anandanagar, Bangalore, India. IEEE ICCIC-2011, Dec $15^{\text {th }}-18^{\text {th }} 2007$,ISBN 978-1-61284-694-1.

[16] Manish Gupta, Govind Sharma, "An Efficient Modified Artificial Bee Colony Algorithm for Job Scheduling Problem”, International Journal of Soft Computing and Engineering (IJSCE) ISSN: 22312307, Volume-1, Is sue-6, January 2012 .

[17] Jan Flusser, Filip Sroubek, and Barbara Zitov, "Image Fusion: Principles, Methods, and Applications", Tutorial EUSIPCO 2007. 


\section{Author's Profile}

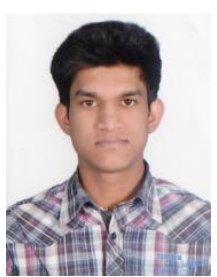

Prabhat Kumar Sharma was born in Jammu City, Jammu and Kashmir State, India Country in 19th Sept 1990. Completed his B.E. from Reva Institute of Technology and Management, Bangalore, India in 2012. He is currently working as Engineer at Tata Elxsi, Bangalore, India since Ju ly 2012.

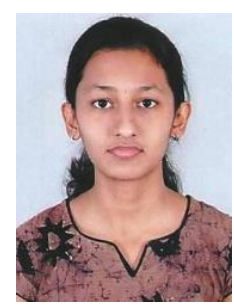

Bhavya V.S. was born in Bangalore City, Karnataka State, India Country on 19th December 1990. Completed her B.E. from Reva Institute of Technology and Management, Bangalore, India in 2012.

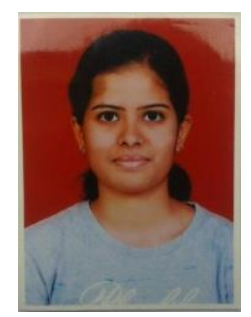

Navyashree $\mathbf{K} \mathbf{M}$ was born in Davangere City, Karnataka State, India Country on 2nd Feb 1991.Completed B.E. from Reva Institute of Technology and Management, Bangalore, India in 2012. She is currently working as Associate Consultant at Oracle Financial Services Software Limited Bangalore, India since August 2012.

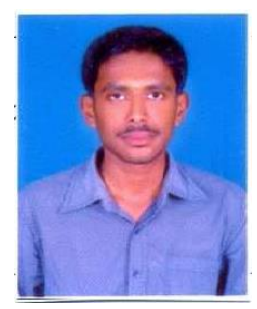

Sunil K.S was born in Bangalore City, Karnataka State, India Country in 12th Ju ly 1990. Completed his B.E. from Reva Institute of Technology and Management, Bangalore, India in 2012. He is currently working as associate consultant at Oracle Financial Services Software Limited Bangalore, India since August 2012.

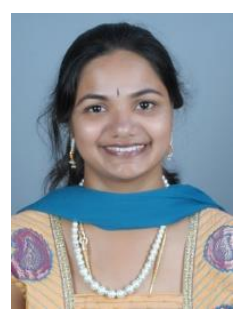

Pavithra $\mathbf{P}$ was born in Hiriyur Town, Karnataka State, India Country on 20th March 1986. Completed her B.E.(ISE) from Sri Venkateshwara College of Engineering, Bangalore, India in 2008 and M.Tech(CSE) from Nitte Minakshi Institute of Technology, Bangalore, India in 2010. She is currently working as Asst. Prof in Reva Institute of Technology and Management, Bangalore, India.

How to cite this paper: Prabhat Kumar Sharma, Bhavy a V S, Navyashree K M, Sunil K S, Pavithra P,"Artificial Bee
Colony and Its Application for Image Fusion", International Journal of Information Technology and Computer Science(IJITCS), vol.4, no.11, pp.42-49, 2012. DOI: 10.5815/ijitcs.2012.11.06 